International Journal of Wireless \& Mobile Networks (IJWMN) Vol. 4, No. 6, December 2012

\title{
Weighted Dynamic Distributed Clustering PROTOCOL FOR HETEROGENEOUS WIRELESS SENSOR NETWORK
}

\author{
Said Benkirane ${ }^{1}$, Abderrahim Beni hssane ${ }^{1}$, Moulay Lahcen Hasnaoui ${ }^{2}$, Mostafa \\ Saadi $^{1}$ and Mohamed Laghdir ${ }^{1}$ \\ ${ }^{1}$ MATIC laboratory, Department of Mathematics and Computer Science, \\ Chouaïb Doukkali University, Faculty of Sciences El Jadida, Morocco \\ sabenk1@hotmail.com, abenihssane@yahoo.fr, saadi_mo@yahoo.fr, \\ laghdirmeyahoo.fr \\ ${ }^{2}$ Computer Science Department, Faculty of Sciences Dhar el Mahraz, \\ Sidi Mohammed Ben Abdellah University, Fez, Morocco. \\ mlhnet2002@yahoo.ca
}

\begin{abstract}
In wireless sensor networks (WSN), conserving energy and increasing lifetime of the network are a critical issue that has been addressed by substantial research works. The clustering technique has been proven particularly energy-efficient in WSN. The nodes form groups (clusters) that include one cluster head and member clusters. Cluster heads (CHs) are able to process, filter, gather the data sent by sensors belonging to their cluster and send it to the base station. Many routing protocols which have been proposed are based on heterogeneity and use the clustering scheme such as SEP and DEEC.

In this paper we introduce a new approach called WDDC in which cluster heads are chosen on the basis of probability of ratio of residual energy and average energy of the network. It also takes into consideration distances between nodes and the base station to favor near nodes with more energy to be cluster heads. Furthermore, WDDC is dynamic; it divides network lifetime in two zones in which it changes its behavior.

Simulation results show that our approach performs better than the other distributed clustering protocols such as SEP and DEEC in terms of energy efficiency and lifetime of the network.
\end{abstract}

\section{KEYWORDS:}

Wireless Sensor Networks, Energy Efficiency, Dynamic Protocols, Heterogeneous Network, Clustering.

\section{INTRODUCTION}

A Wireless Sensor Network (WSN) is composed of hundreds or thousands of sensor nodes. These small sensor nodes contain sensing, data processing and communicating components. A wireless sensor network comprises a base station (BS) that can communicate with a number of wireless sensors via radio link.

The base station in sensor networks is very often a node with high processing power, high storage capacity and the battery used can be rechargeable.

Data are collected at a sensor node and transmitted to the BS directly or by means of other nodes. All collected data for a specific parameter like temperature, pressure, humidity, etc are processed in the BS and then the expected amount of the parameter will be estimated. In these networks, the position of sensor nodes need not be engineered or pre-determined, which allows random deployment in inaccessible terrains or disaster relief operations [1].

Communication protocols highly affect the performance of WSNs by evenly distributing energy load and decreasing their energy consumption and thereupon prolonging their lifetime. Thus, developing energy-efficient protocols is fundamental for prolonging the lifetime of WSNs [2]. DOI : $10.5121 /$ ijwmn.2012.4606 
International Journal of Wireless \& Mobile Networks (IJWMN) Vol. 4, No. 6, December 2012

Among the proposed communication protocols, hierarchical (cluster based) ones have significant savings in the total energy consumption of wireless micro sensor network [3][4][5]. In these protocols, the sensor nodes are grouped into a set of disjoint clusters. Each cluster has a designated leader, the so-called cluster-head $(\mathrm{CH})$. Nodes in one cluster do not transmit their gathered data directly to the BS, but only to their respective cluster-head.

Besides, it is approved [3] that the use of the clustering technique reduces communication energy more than direct transmission (DT) and minimum transmission-energy (MTE) routing.

In this paper, we propose a new approach, called WDDC, which is based on the ratio of residual energy and average energy of the network and takes into consideration distances between nodes and the base station to determine near nodes and distant nodes in order to give more chance to the nearest nodes to be cluster heads by modifying the election probability value for every type of nodes.

WDDC is dynamic, autonomous and more energy-efficient. Simulation results show that it prolongs the network lifetime much more significantly than the other clustering protocols such as SEP and DEEC.

The remainder of the paper is organized as follows: Section 2 contains the related work done. Section 3 explains the heterogeneous network and radio energy dissipation model. Section 4 describes the DEEC protocol followed by section 5 which describes our WDDC approach. Section 6 shows the simulation results and finally Section 7 gives concluding remarks.

\section{RELATED WORK}

There are two kinds of clustering schemes. The first kind is called homogeneous clustering protocols. They are applied in homogeneous networks, where all nodes have the same initial energy, such as LEACH [3], PEGASIS [6], and HEED [7]. The second kind of clustering algorithms applied in heterogeneous networks are referred to as heterogeneous clustering schemes [8], where all the nodes of the sensor network are equipped with different amounts of energy, such as SEP [9], M-LEACH [10], EECS [11], LEACH-B [12] and DEEC [13].

WSNs are more likely to be heterogeneous networks than homogeneous ones. Thus, the protocols should be fit for the characteristic of heterogeneous wireless sensor networks. Moreover, in $[14,15]$, they propose protocols which use a new conception based on the energy left in the network.

Low-Energy Adaptive Clustering Hierarchy (LEACH) [3] is proposed by Heinzelman et al., which is one of the most fundamental protocol frameworks in the literature. LEACH is a clustering-based protocol architecture which utilizes randomized rotation of the Cluster-Heads $(\mathrm{CHs})$ to uniformly distribute the energy budget across the network. The sensor nodes are arranged into several clusters and in each cluster, one of the sensor nodes is chosen to be $\mathrm{CH}$. Each node will transmit its data to its own $\mathrm{CH}$ which forwards the sensed data to the BS finally. Both the communication between sensor nodes and $\mathrm{CH}$ and that between $\mathrm{CHs}$ and the BS are direct, single-hop transmission.

PEGASIS [6] is a chain-based protocol which evades cluster formation and uses only one node in a chain to transmit to the BS instead of using multiple nodes. Heinzelman, et.al. [14] proposed LEACH-centralized (LEACH-C), a protocol that employs a centralized clustering algorithm and the same steady-state protocol as LEACH. O. Younis, et al. [7] proposed HEED (Hybrid Energy-Efficient Distributed clustering), which regularly select cluster heads according to a hybrid of the node residual energy and a secondary parameter, such as node proximity to its neighbors or node degree. G. Smaragdakis, I. Matta and A. Bestavros proposed SEP (Stable Election Protocol) [9] in which every sensor node in a heterogeneous two-level hierarchical 
International Journal of Wireless \& Mobile Networks (IJWMN) Vol. 4, No. 6, December 2012

network independently elects itself as a cluster head based on its initial energy relative to that of other nodes. Li Qing et.al [13] proposed DEEC (Distributed Energy Efficient Clustering) algorithm in which the cluster head is selected on the basis of probability of ratio of residual energy of each node and the average energy of the network. In this algorithm, a node having more energy has more chances to be a cluster head. This solution doesn't take into account the notion of distances between nodes and the base station, and this protocol is not dynamic.

Among the new researches, we can mention the work done by Latif et al. [16]. In this work, the authors compared four protocols using linear programming formulation technique. It is concluded from their analytical simulation results that DEEC is the most energy-efficient protocol for heterogeneous node energy network.

Another work made by Chang et al. [17] proposes an energy-saving clustering algorithm to provide efficient energy consumption in the network. The approach proposed in this work is to reduce data transmission distance of sensor nodes in wireless sensor networks by using the uniform cluster notion. In order to make an ideal distribution for sensor node clusters, they calculate the average distance between the sensor nodes and take into account the residual energy for selecting the appropriate cluster head nodes. The lifetime of wireless sensor networks is extended by using the uniform cluster location and equalizing the network loading among the clusters.

There is also another work by Rajni et al. [18] in which the authors propose a new clustering protocol for prolonging the network lifetime. The algorithm proposed is the modification of DEEC protocol. It is called a Clustering Technique for Routing in Wireless Sensor Networks (CTRWSN). It is a self-organizing and dynamic clustering method that divides dynamically the network on a number of clusters previously fixed. The operation of CTRWSN is divided into rounds where each round consists of a clustering stage and distributed multi-hop routing stage.

\section{Heterogeneous Network and Radio Energy Dissipation MODEL}

\subsection{Heterogeneous Network Model}

In this study, we explain the network model. We suppose that there are $\mathrm{N}$ sensor nodes, which are uniformly dispersed within a $\mathrm{M} \times \mathrm{M}$ square region (Figure.1). The nodes always have data to transmit to a base station, which is often far from the sensing area. The network is structured into a clustering hierarchy, and the cluster-heads execute fusion function to reduce correlated data produced by the sensor nodes within the clusters. The cluster-heads transmit the aggregated data directly to the base station. We assume that the nodes are static (not moving). In the twolevel heterogeneous networks, there are two types of sensor nodes, i.e., the advanced nodes and normal nodes. Note $E_{0}$ the initial energy of the normal nodes, and $m$ the fraction of the advanced nodes, which own a times more energy than the normal ones. Thus there are $\mathrm{Nm}$ advanced nodes equipped with initial energy of $E_{0}(1+a)$, and $N(1-m)$ normal nodes equipped with initial energy of $E_{0}$. The total initial energy of the two-level heterogeneous networks is given by:

$$
E_{\text {total }}=N(1-m) E_{0}+N m E_{0}(1+a)=N E_{0}(1+a m)
$$


International Journal of Wireless \& Mobile Networks (IJWMN) Vol. 4, No. 6, December 2012

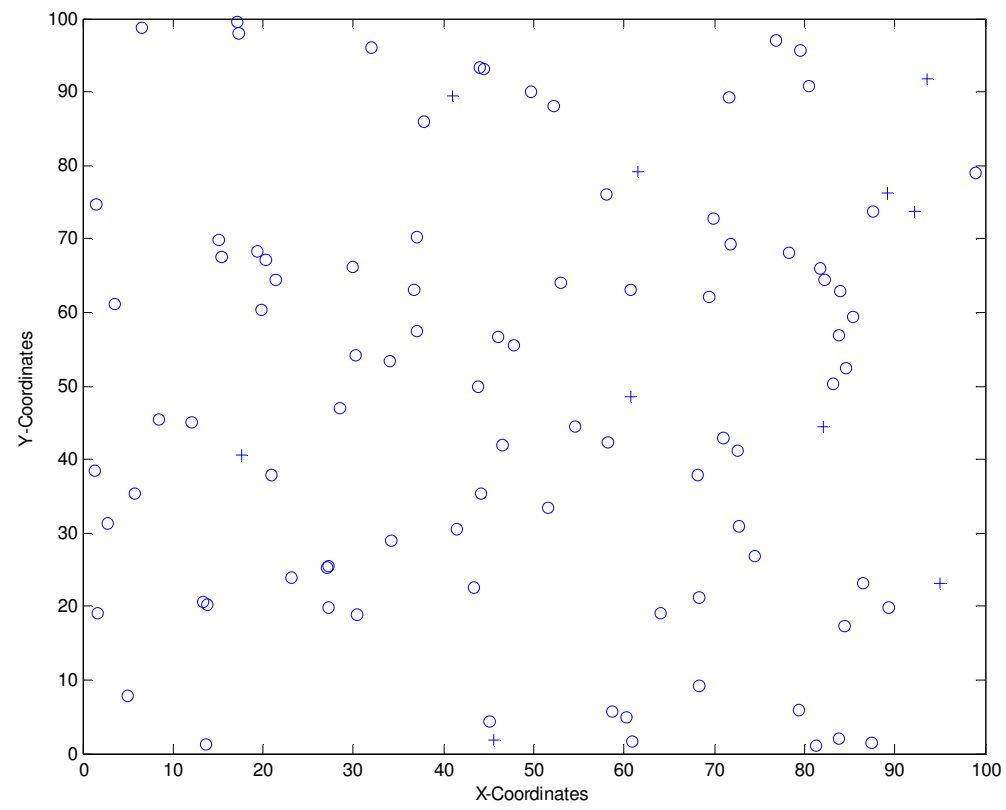

Figure 1.100 nodes randomly deployed in the network (o normal node, + advanced node).

\subsection{Radio Energy Dissipation Model}

According to the radio energy dissipation model proposed in [14] (Figure 2) and in order to achieve an acceptable Signal-to-Noise Ratio (SNR) in transmitting an L-bit message over a distance $d$, the energy expended by the radio is given by:

$$
E_{T x}(l, d)= \begin{cases}l E_{\text {elec }}+l \epsilon_{f s} d^{2}, & d<d_{0} \\ l E_{\text {elec }}+l \epsilon_{m p} d^{4}, & d \geq d_{0}\end{cases}
$$

Where $E_{\text {elec }}$ is the energy dissipated per bit to run the transmitter $E_{T x}$ or the receiver $E_{R x}$ circuit, $\epsilon_{\mathrm{fs}}$ is the free space fading energy, $\epsilon_{\mathrm{mp}}$ is the multi-path fading energy and $\mathrm{d}$ is the distance between the sender and the receiver.

To receive this message the radio expends energy:

$$
E_{R x}(l)=l E_{\text {elec }}
$$

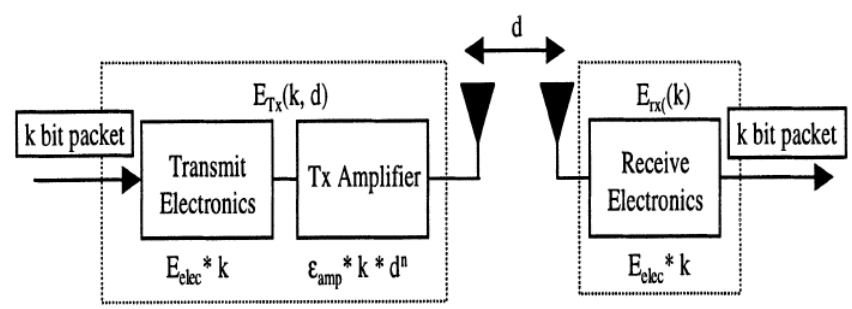

Figure 2. Radio Energy Dissipation Model. 


\section{DEEC PROTOCOL:}

In DEEC [13] the cluster-heads are chosen by a probability based on the ratio between residual energy of each node and the average energy of the network.

As mentioned in section 3.1, the total initial energy of the two-level heterogeneous networks is computed as:

$$
E_{\text {total }}=N(1-m) E_{0}+N m E_{0}(1+a)=N E_{0}(1+a m)
$$

The probability threshold which each node $s_{i}$ uses to determine whether itself to become a cluster-head in each round is as follows:

$$
T\left(s_{i}\right)= \begin{cases}\frac{p_{i}}{1-p_{i}\left(r \bmod \frac{1}{p_{i}}\right)} \text { if } & s_{i} \in G \\ 0 & \text { otherwise }\end{cases}
$$

Where $G$ is the group of nodes that are eligible to be cluster heads at round $r$. In each round $r$, when node $s_{i}$ finds itself eligible to be a cluster head, it will choose a random number between 0 and 1 . If the number is less than threshold $T\left(s_{i}\right)$, the node $s_{i}$ becomes a cluster head during the current round.

Also, for two-level heterogeneous networks, $p_{i}$ is defined as follows:

$$
P_{i}= \begin{cases}\frac{\mathrm{p}_{\mathrm{opt}} \mathrm{E}_{\mathrm{i}}(\mathrm{r})}{(1+\mathrm{a} m) \bar{E}(\mathrm{r})} & \text { if } \mathrm{s}_{\mathrm{i}} \text { is the normal node } \\ \frac{\mathrm{p}_{\mathrm{opt}}(1+\mathrm{a}) \mathrm{E}_{\mathrm{i}}(\mathrm{r})}{(1+\mathrm{a} m) \bar{E}(\mathrm{r})} & \text { if } \mathrm{s}_{\mathrm{i}} \text { is the advanced node }\end{cases}
$$

Where $E_{i}(r)$ denotes the residual energy of node $\mathrm{s}_{\mathrm{i}}$ and $\bar{E}(\mathrm{r})$ is the average energy of the network at round $\mathrm{r}$.

The estimate value of $\bar{E}(\mathrm{r})$ is:

$$
\bar{E}(\mathrm{r})=\frac{1}{N} E_{\text {total }}\left(1-\frac{r}{R}\right)(7)
$$

Where $R$ indicates the total rounds of the network lifetime.

The value of $R$ can be approximated as:

$$
R=\frac{E_{\text {total }}}{E_{\text {Round }}}
$$

Where $E_{\text {Round }}$ denote the total energy dissipated in the network during a round $r$. $E_{\text {Round }}$ is given by:

$$
E_{\text {Round }}=L\left[2 N E_{\text {elec }}+N E_{D A}+k \epsilon_{m p} d_{t o B S}^{4}+N \epsilon_{f s} d_{\text {toCH }}^{2}\right]
$$

Where $k$ is the number of clusters, $\mathrm{E}_{\mathrm{DA}}$ is the data aggregation cost expended in $\mathrm{CH}$ and $\mathrm{BS}$, $d_{t o B S}$ is the average distance between the cluster-head and the base station and $d_{\text {toCH }}$ is the average distance between cluster members and the cluster-head.

According to [14][19] we can get the equations as follows:

$$
d_{\text {toCH }}=\frac{M}{\sqrt{2 k \pi}} \text { And } d_{t o B S}=0.765 \frac{M}{2}
$$


Then the optimal value of $k$ is :

$$
k=\frac{\sqrt{E_{f s}}}{\sqrt{E_{m p}}} \frac{\sqrt{N}}{\sqrt{2 \pi}} \frac{M}{d_{t o B S}^{2}}
$$

Using Eqs. (10) and (11), we can obtain the energy $E_{\text {Round }}$ dissipated during a round and thus we can compute the network lifetime $R$ by Eq. (8).

\section{WDDC PROTOCOL:}

According to the Radio Energy Dissipation Model, the minimum required amplifier energy is proportional to the square of the distance from the transmitter to the destined receiver $\left(T x-A m p l i f i e r \propto d^{2}\right)$ [20]. So the transmission energy consumption will augment greatly as the transmission distance rises. It means that the $\mathrm{CHs}$ far from the BS must use much more energy to transmit the data to the BS than those close to the BS. Therefore, after the network operates for some rounds there will be significant difference between the energy consumption of the nodes near the BS and that of the nodes far from the BS.

In our approach, nodes with more energy than the other nodes and the nodes with less distance from the BS have more chance to be selected as a cluster-head for current round.

For this reason, we introduce new weighted probabilities for every type of nodes according to their residual energy and the average energy of the network in current round. We also take into consideration distances between nodes and the base station in order to favor nodes with more energy and nearest to the BS to become cluster heads.

The new probabilities are as follows:

$$
\begin{gathered}
P_{n r m}=\frac{p_{o p t} E_{i}(r)}{(1+a m) \bar{E}(r)} *(1-w) \\
P_{a d v}=\frac{(1+a) p_{o p t} E_{i}(r)}{(1+a m) \bar{E}(r)} *(1-w)
\end{gathered}
$$

Where $w$ is the weighted factor that contains the notion of distances.

We suppose that after spreading the nodes in network field, the base station broadcasts a "hello" message to all the nodes at a given power level. Each node can compute its estimated distance $\left(D_{i}\right)$ from the BS based on the received signal strength.

The average distance is given by:

$$
D_{\text {avg }}=\frac{1}{N} \sum_{i=1}^{N} D_{i}
$$

The value of $D_{\text {avg }}$ can be approximated as:

Where:

$$
D_{\text {avg }} \simeq d_{t o C H}+d_{t o B S}
$$

- $d_{\text {toCH }}$ The average distance between the node and the associate cluster head (figure 3).

- $\mathrm{d}_{\text {toBs }}$ The average distance between the cluster head and the base station (figure 3). 
International Journal of Wireless \& Mobile Networks (IJWMN) Vol. 4, No. 6, December 2012

We assume that the nodes are uniformly distributed and the BS is located in the center of the field. Thus we can use equations (10) to calculate, $d_{\text {toCH }}, d_{\text {toBs }}$ and finally $D_{\text {avg }}$.

We introduce the notion of far nodes and near nodes. However, nodes situated within the circle of radius equal to $D_{\text {avg }}$ are considered near nodes and nodes situated in outside of the circle are considered far nodes (figure 4).

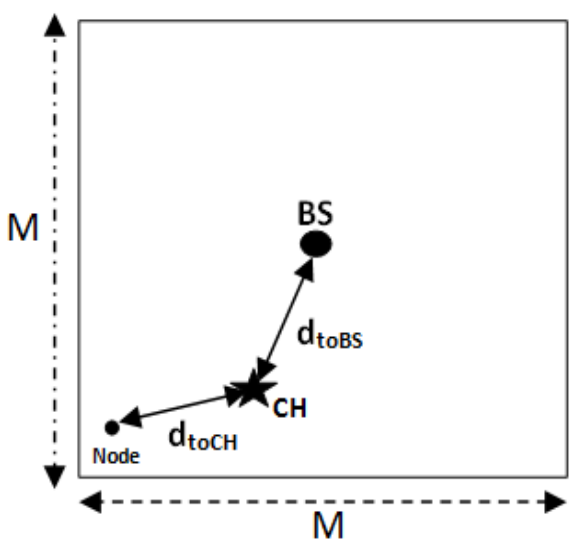

Figure 3. $d_{\text {toCH }}$ and $d_{t o B S}$.

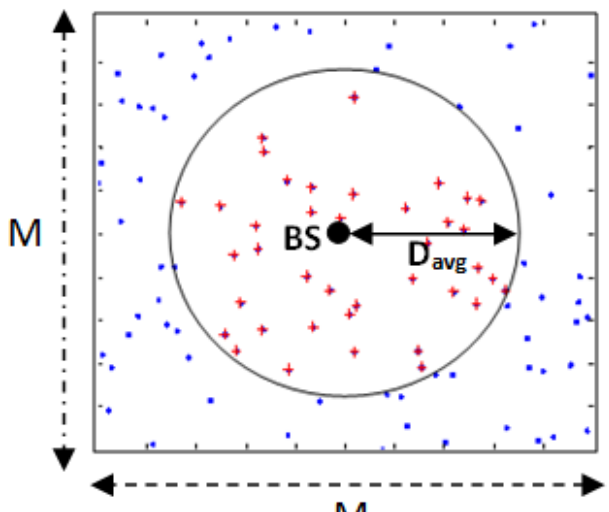

$\mathrm{M}$

Figure 4. Near nodes (+) and far nodes (.)

The surface of the field of the network is obtained by:

$$
A_{1}=M * M
$$

The surface of the circle is given by:

Where:

$$
A_{2}=\Pi * D_{a v g}^{2}
$$

$$
D_{\text {avg }} \simeq d_{t o C H}+d_{t o B S}
$$

Using equations (10) we obtain:

$$
D_{\text {avg }} \simeq \frac{M}{\sqrt{2 k \pi}}+0.765 \frac{M}{2}
$$

The comparison gives:

$$
A_{2}<A_{1}
$$

Finally, the weighted factor $w$ is computed as follows:

$$
w=A_{2} / A_{1}(19)
$$

Furthermore, WDDC is dynamic because it divides network lifetime in two zones, strong zone and normal zone with size $m \times R$ and $R \times(1-m)$ respectively (figure 5), in which it changes its behavior. So, in the first zone only advanced nodes become cluster heads. In the second zone the choice will become normal; it takes into consideration advanced and normal nodes. In both zones we use the new weighted probabilities mentioned in (12) and the probability threshold mentioned in (5). 
International Journal of Wireless \& Mobile Networks (IJWMN) Vol. 4, No. 6, December 2012

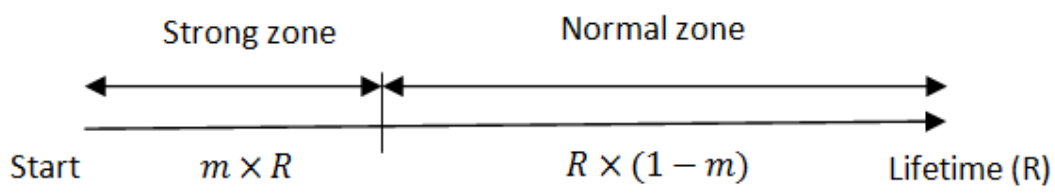

Figure 5. Strong zone and Normal zone

We notice that the size of zones is dynamic. It depends on the value of the fraction of the advanced nodes $(m)$. The strong zone increases when $m$ rises and vice versa for the normal zone; it decreases when $m$ increases (figure 6).

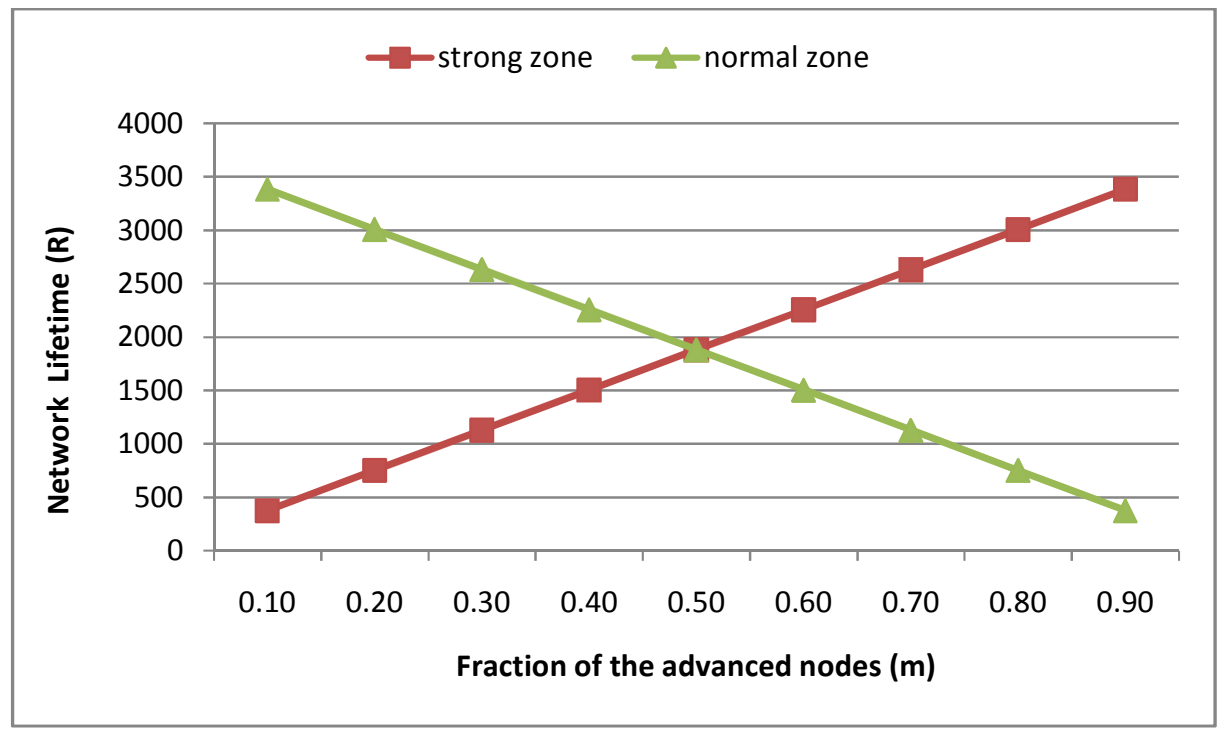

Figure 6. Sizes of strong zone and normal zone.

\section{Communications between Cluster heads and member nodes}

Like LEACH [3], after the cluster-heads are selected, the cluster-heads inform all sensor nodes in the network that they are the new cluster heads. And then, other nodes organize themselves into local clusters by choosing the most appropriate cluster-head (normally the closest clusterhead) (figure.7). Thereafter, the $\mathrm{CH}$ receives sensed data from cluster members according to TDMA schedule that was created and transmitted to them.

\section{Communications between cluster heads and the base station.}

Each node sends its data during their assigned transmission time to the respective associate cluster head. The $\mathrm{CH}$ node must keep its receiver on in order to receive all the data from the nodes in the cluster. When all the data is received, the cluster head node executes signal processing functions to compress the data into a single signal. When this phase is completed, each cluster head can send the combined data to the base station.

The consumed energy of cluster head $\mathrm{CH}_{\mathrm{i}}$ is composed of three parts: data receiving, data aggregation and data transmission. Then:

$$
E\left(C H_{i}\right)=m_{i} l E_{\text {elec }}+\left(m_{i}+1\right) l E_{D A}+l\left(E_{\text {elec }}+\epsilon_{f s} d^{2}\right)(20)
$$


Where: $m_{i}$ is the sum of member nodes in associate cluster and $d=D_{i}$ the distance between $\mathrm{CH}$ and the BS (in this case we consider $<d_{0}$ ) (figure 7).

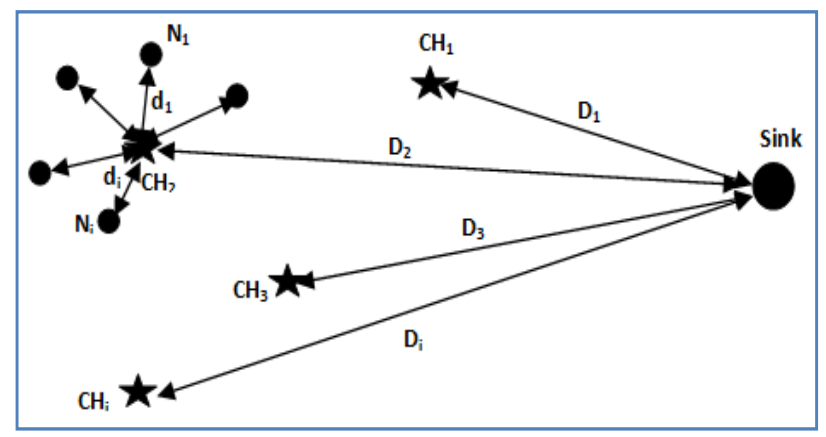

Figure 7. Distances between $\mathrm{CH}$ and the base station

\section{Simulation Results}

We evaluate the performance of WDDC protocol using MATLAB. We consider a wireless sensor network with $\mathrm{N}=100$ nodes randomly distributed in a $100 \mathrm{~m} \times 100 \mathrm{~m}$ field. We assume the base station is in the center of the sensing region. We ignore the effect caused by signal collision and interference in the wireless channel and we have fixed the value of $d_{0}$ at 70 meters like on DEEC.

The radio parameters used in our simulations are shown in Table 1. The protocols compared with WDDC include SEP and DEEC.

Table 1. Radio characteristics used in our simulations

\begin{tabular}{|l|l|}
\hline Parameter & Value \\
\hline$E_{\text {elec }}$ & $5 \mathrm{~nJ} / \mathrm{bit}$ \\
\hline$\epsilon_{f s}$ & $10 \mathrm{pJ} / \mathrm{bit} / \mathrm{m}^{2}$ \\
\hline$\epsilon_{m p}$ & $0.0013 \mathrm{pJ} / \mathrm{bit} / \mathrm{m}^{4}$ \\
\hline$E_{0}$ & $0.5 \mathrm{~J}$ \\
\hline$E_{D A}$ & $5 \mathrm{~nJ} / \mathrm{bit} / \mathrm{message}$ \\
\hline$d_{0}$ & $70 \mathrm{~m}$ \\
\hline Message size & $4000 \mathrm{bits}$ \\
\hline$p_{\text {opt }}$ & 0.1 \\
\hline
\end{tabular}

Due to the heterogeneity factors, $\mathrm{R}$ is taken as $2 \times \mathrm{R}$ (Since $\bar{E}(\mathrm{r})$ will be too large at the end from Eq.(7), thus some nodes will not die finally).

We define stable time as time until the first node dies (FND), and unstable time the time from the fist node dies until the last node dies. In other words, lifetime is the addition of stable time and unstable time.

We define also HNA (half of nodes alive) as the half of the total number of nodes that have not yet expended all their energy. All protocols remain useful during this period but after $50 \%$ of nodes die, the network becomes completely unstable and protocols will become useless. 


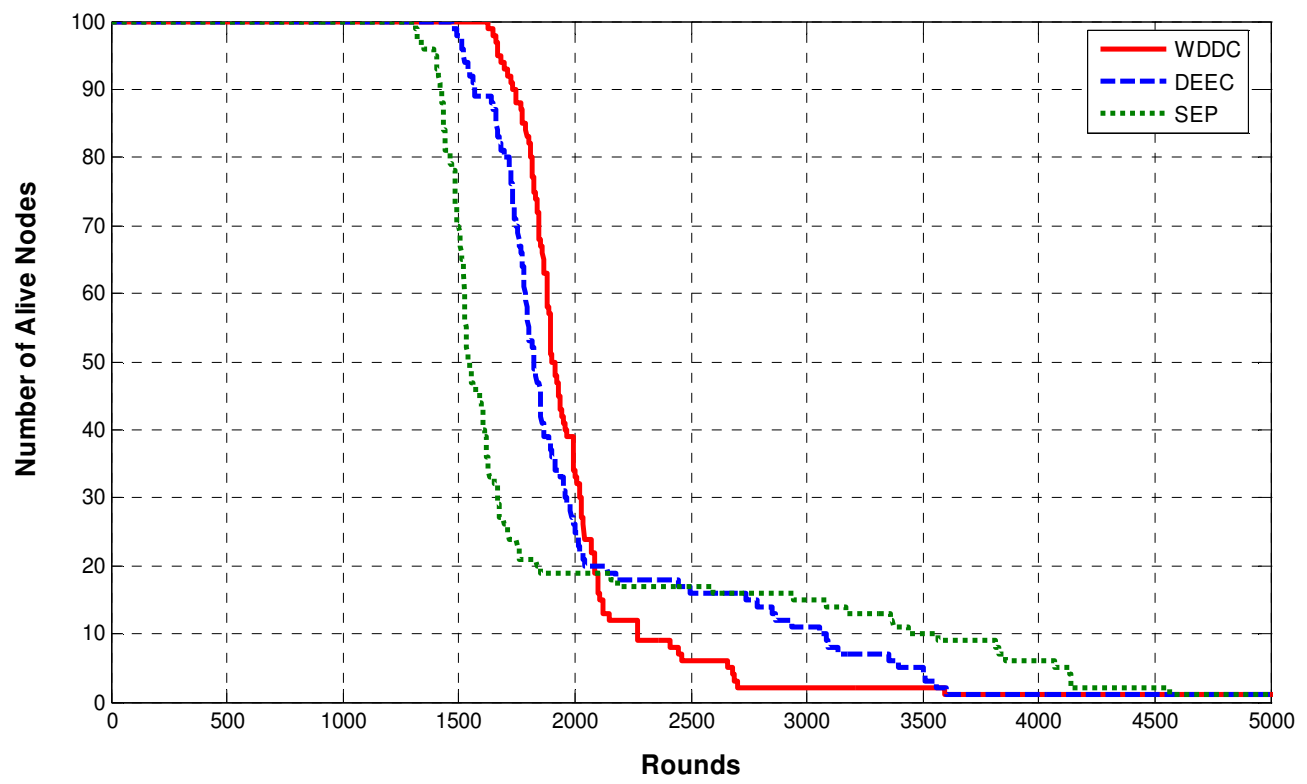

Figure 8 . Number of nodes alive over time $(\mathrm{m}=0.2$ and $\mathrm{a}=3)$

According to the figure 8, we notice that the stable time of WDDC is large compared to that of SEP and DEEC. A longer stable time metric is important because it gives the end user reliable information of the sensing area, which extend the network lifetime. This reliability is vital for sensitive applications like tracking fire in forests.

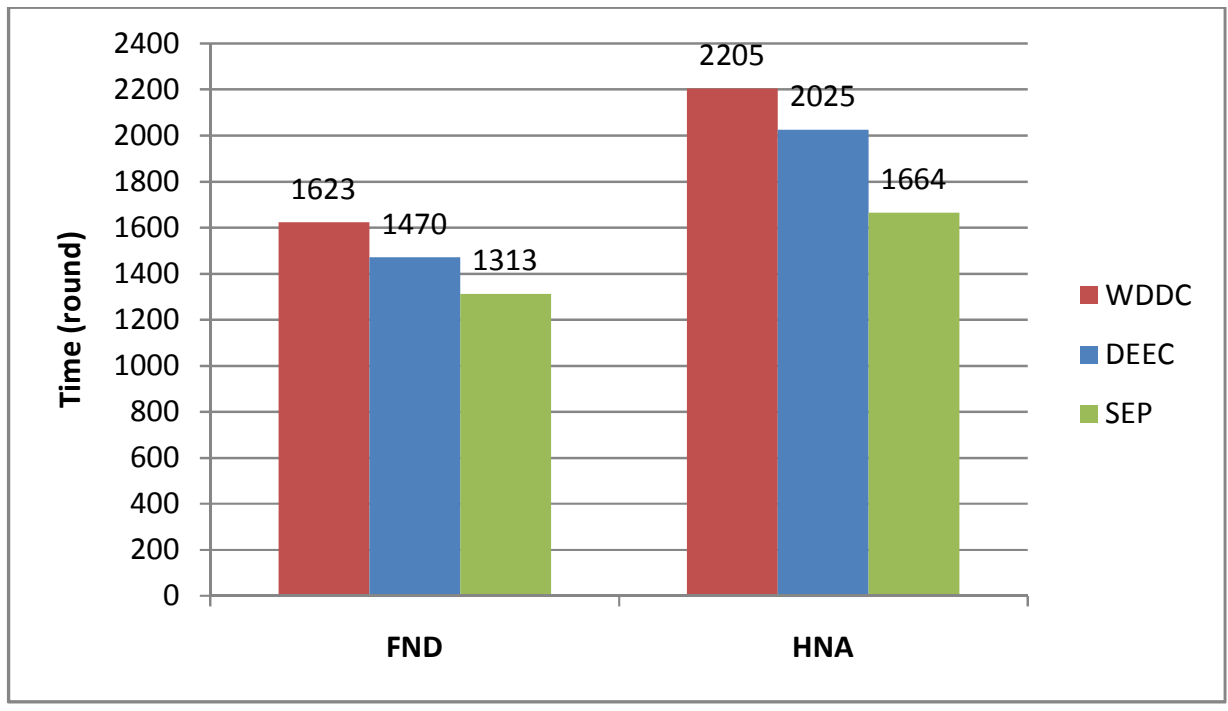

Figure 9. FND and HNA $(m=0.2$ and $a=3)$

Figure 9 shows the comparison between all nodes in terms of FND and HNA when $m=0.2$ and $a=3$. Obviously, we can remark that our protocol WDDC contains a larger period of stability time than SEP and DEEC, which increases the efficiency of the network.

We notice the same results for HNA. Therefore, WDDC performs better than the other simulated protocols. When half the number of nodes have expended all their energy, the network becomes inefficient. 


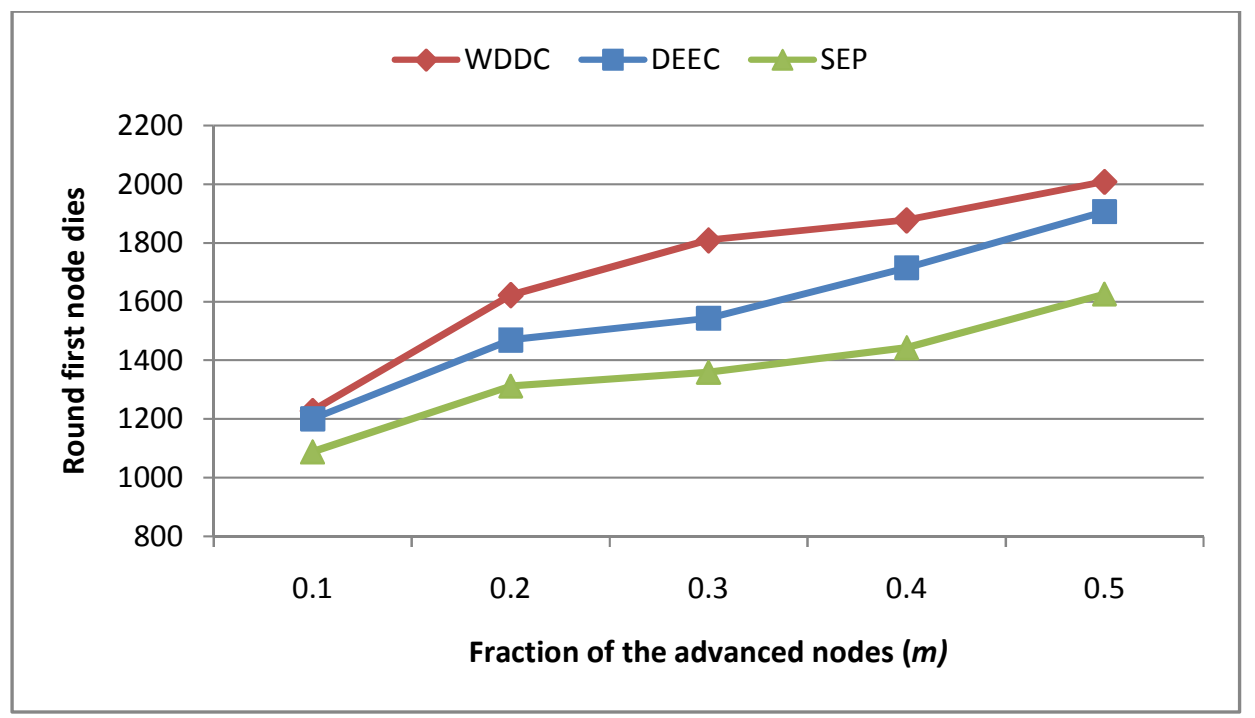

Figure 10. Round first node dies when $m$ is varying.

Second, we run simulation for our proposed protocol WDDC to compute the round of the death of the first node when varying $m$, and we compare the results to SEP and DEEC protocols. We increase the fraction $\mathrm{m}$ of the advanced nodes from 0.1 to 0.5 ; Figure 10 shows the number of round when the first node dies. We observe that WDDC performs better than SEP and DEEC.

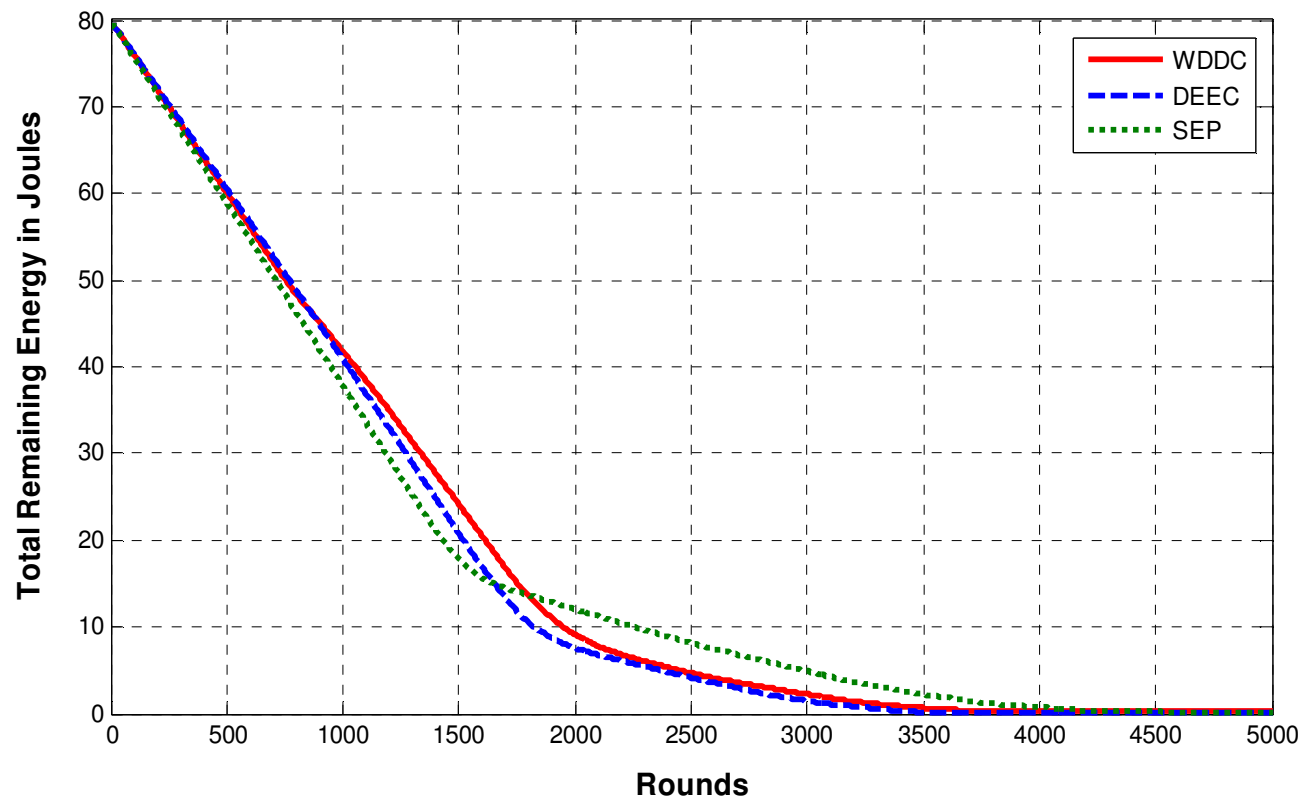

Figure 11. Total remaining energy over time of WDDC, DECC and SEP $(m=0.2$ and $a=3)$

Figure 11 shows the remaining energy over time for all simulated protocols and it reveals that WDDC consumes less energy in comparison to the others, which helps to extend the network lifetime. Here, approximately $7 \%$ of energy is saved at round 1500 . This is because in our 
International Journal of Wireless \& Mobile Networks (IJWMN) Vol. 4, No. 6, December 2012

approach we took into consideration distances between nodes and the base station. Therefore, cluster heads situated far from the base station consume more energy than cluster heads situated near the base station, which saves the total energy of the network.

\section{CONCLUSION}

In this paper we have explained WDDC, a weighted dynamic distributed clustering protocol suitable for heterogeneous wireless sensor networks, and compared it to the SEP and DEEC protocols. WDDC is an energy-aware clustering protocol in which every sensor node separately elects itself as a cluster-head on the basis of probability of ratio of residual energy and average energy of the network and takes into consideration distances between nodes and the base station. Thus, nodes with less energy than the other nodes and the nodes with more distance from the BS have the smallest chance to be selected as a cluster-head for current round.

WDDC is dynamic, autonomous and more energy-efficient; it divides network lifetime in two zones, strong zone and normal zone, in which it changes its behavior so as to be more efficient. WDDC uses the two hierarchical levels concept which offers a better use and optimization of the energy dissipated in the network. Results from our simulations show that WDDC provides better performance for energy efficiency and network lifetime.

\section{REFERENCES}

[1] F. Akyildiz, W. Su, Y. Sankarasubramaniam, and E. Cayirici, “A survey on sensor networks", IEEE communications magazine 40 (8) (2002)102-114.

[2] J. N. Al-Karaki and A. E. Kamal, "Routing Techniques in Wireless Sensor Networks: A Survey", IEEE Journal of Wireless Communications, vol. 11, no. 6, Dec. 2004, pp. 6-28.

[3] W.B. Heinzelman, A.P. Chandrakasan, and H. Balakrishnan, "Energy efficient communication protocol for wireless microsensor networks", in Proceedings of the 33rd Hawaii International Conference on System Sciences (HICSS-33), January 2000.

[4] O. Younis, M. Krunz, and S. Ramasubramanian, "Node Clustering in Wireless Sensor Networks: Recent Developments and Deployment Challenges," IEEE Network (special issue on wireless sensor networking), vol. 20, issue 3, pp. 20-25, May 2006.

[5] A. Abbasi and M. Younis. "A survey on clustering algorithms for wireless sensor networks". Computer Communication, 30(14-15):2826-2841, 2007.

[6] S. Lindsey and C.S. Raghavenda, "PEGASIS: power efficient gathering in sensor information systems”, in Proceeding of the IEEE Aerospace Conference, Big Sky, Montana, March 2002.

[7] O. Younis, and S. Fahmy, "HEED: A hybrid, energy-efficient, distributed clustering approach for ad hoc sensor networks", IEEE Transactions on Mobile Computing 3 (4) (2004) 660-669.

[8] D. Kumar, T. C. Aseri and R.B. Patel, "EEHC: Energy efficient heterogeneous clustered scheme for wireless sensor networks”, Elsevier, Computer Communications 32 (2009) 662-667.

[9] G. Smaragdakis, I. Matta and A. Bestavros, "SEP: A Stable Election Protocol for clustered heterogeneous wireless sensor networks", in Second International Workshop on Sensor and Actor Network Protocols and Applications (SANPA 2004), 2004.

[10] V. Mhatre, and C. Rosenberg, "Design guidelines for wireless sensor networks: communication, clustering and aggregation", Ad Hoc Network Journal 2 (1) (2004) 45-63.

[11] M. Ye, C. Li, G. Chen and J. Wu, "EECS: an energy efficient cluster scheme in wireless sensor networks", in IEEE International Workshop on Strategies for Energy Efficiency in Ad Hoc and Sensor Networks (IEEE IWSEEASN2005), Phoenix, Arizona, April 7-9, 2005.

[12] A. Depedri, A. Zanella and R. Verdone, "An energy efficient protocol for wireless sensor networks", in: Autonomous Intelligent Networks and Systems (AINS 2003), Menlo Park, CA, June 30-July 1, 2003. 
International Journal of Wireless \& Mobile Networks (IJWMN) Vol. 4, No. 6, December 2012

[13] L. Qing, Q. Zhu and M. Wang, "Design of a distributed energy-efficient clustering algorithm for heterogeneous wireless sensor networks". ELSEVIER, Computer Communications 29, pp 2230$2237,2006$.

[14] W. B. Heinzelman, A. P. Chandrakasan and H. BalaKrishnan, "An Application-specific Protocol Architecture for Wireless Microsensor Networks," IEEE Transactions on Wireless Communications, 1, No. 4, pp. 660-670, 2002.

[15] V. Loscri, G. Morabito and S. Marano, "A Two-Levels Hierarchy for Low- Energy Adaptive Clustering Hierarchy (TL-LEACH)”. In 0-7803-9152-7/05/20.00 2005 IEEE.

[16] K. Latif, M. Jaffar, N. Javaid, M. N. Saqib, U. Qasim and Z. A. Khan. "Performance Analysis of Hierarchical Routing Protocols in Wireless Sensor Networks" NGWMN with 7th IEEE International Conference on Broadband and Wireless Computing, Communication and Applications (BWCCA 2012), Victoria, Canada, 2012.

[17] J. Y. Chang and P. H. Ju "An efficient cluster-based power saving scheme for wireless sensor networks”. EURASIP Journal on Wireless Communications and Networking 2012 2012:172.

[18] R. Meelu and R. Anand "Performance Evaluation Of Cluster-Based Routing Protocols Used In Heterogeneous Wireless Sensor Networks" International Journal of Information Technology and Knowledge Management. Volume 4, No. 1, pp. 227-231, January-June 2011.

[19] S. Bandyopadhyay and E.J. Coyle, "An Energy Efficient Hierarchical Clustering Algorithm for Wireless Sensor Networks" in: Proceeding of INFOCOM 2003, April 2003.

[20] A. Khadivi and M. Shiva, "FTPASC: A Fault Tolerant Power Aware Protocol with Static Clustering for Wireless Sensor Networks", Proc. of IEEE Int. Conf. on Wireless and Mobile Computing, Networking and Communications, Montreal, Canada, Jun. 2006, pp. 397-401.

\section{Authors:}

Said BENKIRANE: Obtained his Certificate in telecommunications engineering at the National Institute of Posts and Telecommunications, Rabat, Morocco, in 2004, and his M.Sc. degree in computer engineering and network from the University of Sidi Mohammed Ben Abdellah Fez, Morocco in 2006. He has been working as professor of Computer Sciences in high school since 2007, in El Jadida, Morocco. $\mathrm{He}$ is a member of a research group e-NGN (e-Next Generation Networks) for Africa and Middle East. Currently, he is pursuing his Ph.D at the Faculty of Sciences, Chouaib Doukkali University, El Jadida, Morocco. His main research areas include wireless and mobile computing and mobile telecommunications systems.

Abderrahim BENI HSSANE: Is a research and an assistant professor at Science Faculty, Chouaîb Doukkali University, El Jadida, Morocco, since September 1994. He got his B.Sc. degree in applied mathematics and his Doctorate of High Study Degree in computer science, respectively, in 1992 and 1997 from Mohamed V University, Rabat, Morocco. His research interests focus on performance evaluation in wireless networks.

Dr. Moulay Lahcen HASNAOUI: Received his Ph.D in modelling and simulation of semiconductor devices at the Paris-Sud University, France (1991-1995). He worked as research associate in developing fuel cell at Department of Engineering Physics, Polytechnic School, Montreal, Canada (1996-1996). He earned his bachelor's degree in Computer Science from University of Montreal, Canada (19982002). Self-employed as a software developer (2002-2004). He worked as research assistant professor at Mathematics and Computer Science Department at the Faculty
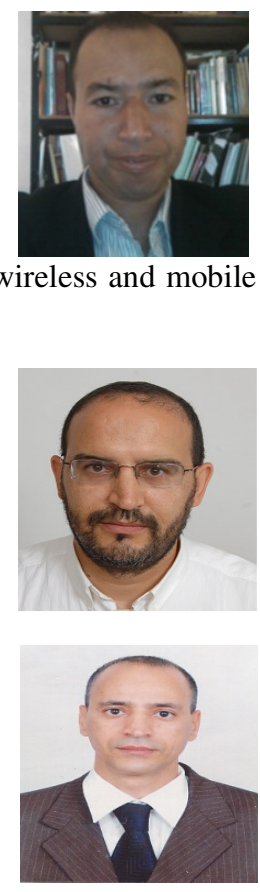
of Sciences, MATIC Laboratory, El Jadida, Morocco, between 2004-2011. He is working as research assistant professor at Computer Sciences Department at the Faculty of Sciences Dhar Al Mahraz, Fez (2011). 
International Journal of Wireless \& Mobile Networks (IJWMN) Vol. 4, No. 6, December 2012

Mostafa SAADI : Received the B.Sc. degree in Computer Sciences at the University Hassan $2^{\text {nd }}$, Faculty of Sciences Ain-Chook, Casablanca, Morocco, in 2003, and a M.Sc. degree in Mathematical and Computer engineering at the University Chouaib Doukkali, Faculty of Sciences, El Jadida (FSJ), Morocco, in 2009. He has been working as a professor of Computer Sciences in high school since 2003, in Sidi Rahal Beach, Morocco. Currently, he is working toward his Ph.D. at FSJ. His current research interests performance evaluation, analysis and simulation of Wireless Sensor networks.

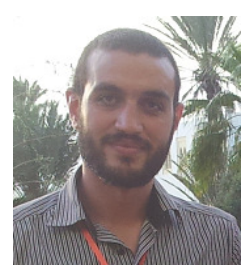

